\title{
NOTE ON ARITHMETIC CONVOLUTION EQUATIONS
}

\author{
MICHAL FEČKAN
}

(Communicated by Jonathan M. Borwein)

Abstract. We investigate the solvability of polynomial equations on the $\mathbb{C}$ algebra of arithmetic functions $g: \mathbb{N} \rightarrow \mathbb{C}$.

\section{INTRODUCTION}

Let $\mathcal{A}$ be the set of all arithmetic functions $g: \mathbb{N} \rightarrow \mathbb{C}$ under point-wise addition and scalar multiplication [5]. The null element is the null function. The Dirichlet convolution $*: \mathcal{A} \times \mathcal{A} \rightarrow \mathcal{A}$ is defined by

$$
(g * h)(n)=\sum_{n_{1} n_{2}=n} g\left(n_{1}\right) g\left(n_{2}\right) \quad \forall n \in \mathbb{N} .
$$

Then $\mathcal{A}$ is considered as a $\mathbb{C}$-algebra under linear operations and the Dirichlet convolution.

Let $r \in \mathbb{R}$ and $\mathcal{A}_{r}$ be the complex Banach algebra of arithmetic functions $g \in \mathcal{A}$ endowed with the norm

$$
\|g\|_{r}:=\sum_{n=1}^{\infty}|g(n)| n^{-r} .
$$

For abbreviation we write $g^{* d}:=g * \cdots * g$. The following result is proved by Glöckner, Lucht and Porubský in [5]:

Theorem 1.1. For $d \in \mathbb{N}$ and $g \in \mathcal{A}$, let $T: \mathcal{A} \rightarrow \mathcal{A}$ be defined by

$$
T g:=a_{d} * g^{* d}+a_{d-1} * g^{*(d-1)}+\cdots+a_{1} * g+a_{0}
$$

with $a_{d}, a_{d-1}, \ldots, a_{1}, a_{0} \in \mathcal{A}$ and $a_{d} \neq 0$. If $z_{0}$ is a simple zero of the polynomial

$$
f(z):=a_{d}(1) z^{d}+a_{d-1}(1) z^{(d-1)}+\cdots+a_{1}(1) z+a_{0}(1),
$$

then there exists a uniquely determined solution $g \in \mathcal{A}$ of the equation

$$
T g=0
$$

satisfying $g(1)=z_{0}$. Moreover, if $a_{d}, a_{d-1}, \ldots, a_{1}, a_{0} \in \mathcal{A}_{\rho}$ for some $\rho \geq 0$, then there is an $r \geq \rho$ such that $g \in \mathcal{A}_{r}$.

Received by the editors December 1, 2006.

2000 Mathematics Subject Classification. Primary 11A25.

Key words and phrases. Arithmetic functions, Dirichlet convolution.

This work was supported by the Grant VEGA-SAV 2/7140/27.

(c) 2007 American Mathematical Society Reverts to public domain 28 years from publication 
We refer the reader to papers of Carroll and Gioia [2], Cohen [3], Dehaye [4], Glöckner, Lucht and Porubský [5], Haukkanen [6], Porubský [8] and Subbarao [9] for more details and known results about arithmetic convolution equations.

Theorem 1.1 is proved in [5] by using a deep result from the theory of topological vector spaces: the implicit function theorem in a version of Biller [1, Theorem 7.2]. The main purpose of this note is to present an elementary proof of Theorem 1.1. But we also extend it to any $\rho \in \mathbb{R}$ along with finding a lower bound $r_{0}$ for the number $r$ and with estimating from above the norm $\|g\|_{r_{0}}$ of the solution $g$. At the end of this note, we also solve a simple quadratic convolution equation to apply our general result.

Finally, I thank Professor Štefan Porubský for stimulating discussions during the preparation of this note. I am also grateful to the referee for valuable comments and suggestions which substantially improved the paper.

\section{MAIN RESUltS}

Let $z_{0}$ be a simple zero of (1.1). As in [5], we start from (1.2) with $g(1)=z_{0}$, which has the form

$$
\sum_{j=0}^{d} \sum_{l n_{1} \cdots n_{j}=n} a_{j}(l) g\left(n_{1}\right) \cdots g\left(n_{j}\right)=0 \quad \forall n \in \mathbb{N} .
$$

Next, we rewrite (2.1) as

$$
f^{\prime}(g(1)) g(n)=-\sum_{j=0}^{d} \sum_{\substack{l n_{1} \cdots n_{j}=n \\ n_{1}, \cdots, n_{j}<n}} a_{j}(l) g\left(n_{1}\right) \cdots g\left(n_{j}\right), \quad n \geq 2 .
$$

Since $f^{\prime}(g(1)) \neq 0$, we can solve (2.2) to get the solution $g \in \mathcal{A}$ of (1.2). If in addition $a_{d}, a_{d-1}, \ldots, a_{1}, a_{0} \in \mathcal{A}_{\rho}$ for some $\rho \in \mathbb{R}$, then from (2.2) for $r \geq \rho$ we derive

$$
\begin{aligned}
f^{\prime}(g(1)) g(n) n^{-r}= & -\sum_{j=0}^{d} a_{j}(1) \sum_{\substack{n_{1} \cdots n_{j}=n \\
n_{1}, \ldots, n_{j}<n}} g\left(n_{1}\right) n_{1}^{-r} \cdots g\left(n_{j}\right) n_{j}^{-r} \\
& -\sum_{j=0}^{d} \sum_{\substack{l n_{1} \cdots, n_{j}=n \\
2 \leq l ; n_{1}, \ldots, n_{j}<n\\
}} \times l^{\rho-r} a_{j}(l) l^{-\rho} g\left(n_{1}\right) n_{1}^{-r} \cdots g\left(n_{j}\right) n_{j}^{-r}, \quad n \geq 2 .
\end{aligned}
$$


Hence for $m \geq 3$ we obtain

$$
\begin{aligned}
& \left|f^{\prime}(g(1))\right| \sum_{n=2}^{m}|g(n)| n^{-r} \\
& \leq \sum_{n=2}^{m} \sum_{j=2}^{d}\left|a_{j}(1)\right| \sum_{\substack{n_{1} \cdots n_{j}=n \\
n_{1}, \ldots, n_{j}<n}}\left|g\left(n_{1}\right)\right| n_{1}^{-r} \cdots\left|g\left(n_{j}\right)\right| n_{j}^{-r} \\
& +2^{\rho-r} \sum_{n=2}^{m} \sum_{j=0}^{d} \sum_{\substack{l n_{1} \cdots n_{j}=n \\
2 \leq l ; n_{1}, \ldots, n_{j}<n}}\left|a_{j}(l)\right| l^{-\rho}\left|g\left(n_{1}\right)\right| n_{1}^{-r} \cdots\left|g\left(n_{j}\right)\right| n_{j}^{-r} \\
& \leq \sum_{n=2}^{m} \sum_{j=2}^{d}\left|a_{j}(1)\right| \sum_{k=0}^{j-2}\left(\begin{array}{c}
j \\
k
\end{array}\right)|g(1)|^{k} \sum_{\substack{n_{1} \cdots n_{j-k}=n \\
2 \leq n_{1}, \ldots, n_{j-k}<n}} \\
& \times\left|g\left(n_{1}\right)\right| n_{1}^{-r} \cdots\left|g\left(n_{j-k}\right)\right| n_{j-k}^{-r} \\
& +2^{\rho-r} \sum_{j=0}^{d}\left(\sum_{l=1}^{m}\left|a_{j}(l)\right| l^{-\rho}\right)\left(\sum_{n=1}^{m-1}|g(n)| n^{-r}\right)^{j} \\
& \leq \sum_{j=2}^{d}\left|a_{j}(1)\right| \sum_{k=0}^{j-2}\left(\begin{array}{l}
j \\
k
\end{array}\right)|g(1)|^{k}\left(\sum_{n=2}^{m-1}|g(n)| n^{-r}\right)^{j-k} \\
& +2^{\rho-r} \sum_{j=0}^{d}\left\|a_{j}\right\|_{\rho}\left(\sum_{n=1}^{m-1}|g(n)| n^{-r}\right)^{j} \\
& =\left(\sum_{n=2}^{m-1}|g(n)| n^{-r}\right)^{2}\left(\sum_{j=2}^{d}\left|a_{j}(1)\right| \sum_{k=0}^{j-2}\left(\begin{array}{l}
j \\
k
\end{array}\right)|g(1)|^{k}\left(\sum_{n=2}^{m-1}|g(n)| n^{-r}\right)^{j-2-k}\right) \\
& +2^{\rho-r} \sum_{j=0}^{d}\left\|a_{j}\right\|_{\rho}\left(\sum_{n=1}^{m-1}|g(n)| n^{-r}\right)^{j} .
\end{aligned}
$$

Recalling $g(1)=z_{0}$ and setting

$$
\begin{aligned}
& S_{m}:=\sum_{n=2}^{m}|g(n)| n^{-r}, \quad m \geq 2, \\
& P(z):=\frac{1}{\left|f^{\prime}\left(z_{0}\right)\right|} z^{2}\left(\sum_{j=2}^{d}\left|a_{j}(1)\right| \sum_{k=0}^{j-2}\left(\begin{array}{l}
j \\
k
\end{array}\right)\left|z_{0}\right|^{k} z^{j-2-k}\right), \\
& Q(z):=\frac{1}{\left|f^{\prime}\left(z_{0}\right)\right|} \sum_{j=0}^{d}\left\|a_{j}\right\|_{\rho} z^{j},
\end{aligned}
$$

we obtain

$$
\begin{aligned}
& S_{2}=|g(2)| 2^{-r}, \\
& S_{m} \leq P\left(S_{m-1}\right)+2^{\rho-r} Q\left(\left|z_{0}\right|+S_{m-1}\right), \quad m \geq 3 .
\end{aligned}
$$

If there are $x_{0}>0$ and $r \geq \rho$ such that

$$
\begin{gathered}
|g(2)| 2^{-r} \leq x_{0}, \\
P\left(x_{0}\right)+2^{\rho-r} Q\left(\left|z_{0}\right|+x_{0}\right) \leq x_{0},
\end{gathered}
$$

then clearly

$$
S_{m} \leq x_{0} \quad \forall m \geq 2 .
$$


From (2.5) we derive

$$
2^{\rho-r} \leq \frac{x_{0}-P\left(x_{0}\right)}{Q\left(\left|z_{0}\right|+x_{0}\right)} .
$$

Since $P(0)=P^{\prime}(0)=0$ and $P(z) \geq 0$ for $z \geq 0$, we can easily check that for $d \geq 2$ there are positive constants $M_{0}$ and $x_{0}$ such that

$$
M_{0}:=\max _{z \in[0, \infty)} \frac{z-P(z)}{Q\left(\left|z_{0}\right|+z\right)}=\frac{x_{0}-P\left(x_{0}\right)}{Q\left(\left|z_{0}\right|+x_{0}\right)} .
$$

Then conditions (2.4) and (2.5) hold with these positive constants $M_{0}$ and $x_{0}$ of (2.7) for any $r \geq r_{0}$ where $r_{0}$ is given by

$$
r_{0}:=\max \left\{\frac{\log \left(|g(2)| / x_{0}\right)}{\log 2}, \rho-\min \left\{0, \frac{\log M_{0}}{\log 2}\right\}\right\} .
$$

Here we set $\log 0=-\infty$. We note

$$
g(2)=-\frac{1}{f^{\prime}\left(z_{0}\right)} \sum_{j=0}^{d} a_{j}(2) z_{0}^{j} .
$$

Summarizing, we obtain the following result.

Theorem 2.1. Let $d \geq 2$ and $z_{0}$ be a simple zero of the polynomial (1.1) with $a_{d}, a_{d-1}, \ldots, a_{1}, a_{0} \in \mathcal{A}_{\rho}$ for some $\rho \in \mathbb{R}$ and $a_{d} \neq 0$. Then a unique solution $g \in \mathcal{A}$ of (1.2) satisfying $g(1)=z_{0}$ belongs to $g \in \mathcal{A}_{r_{0}}$ for $r_{0} \geq \rho$ given by (2.8), while the involved constants $x_{0}, M_{0}$ and $g(2)$ of the formula (2.8) are determined by (2.7) and (2.9), respectively. Moreover, we have $\|g\|_{r_{0}} \leq\left|z_{0}\right|+x_{0}$.

To proceed with the investigation of (1.2), we need the following definition.

Definition 2.1. An $a \in \mathcal{A}$ is said to be a constant coefficient if $a(j)=0 \forall j \geq 2$.

Remark 2.1. If not all $a_{j} \in \mathcal{A}, j=0,1, \cdots, d-1, d$, are constant coefficients, then the polynomial $Q(z)$ can be replaced in Theorem 2.1 with the following:

$$
Q(z):=\frac{1}{\left|f^{\prime}\left(z_{0}\right)\right|} \sum_{j=0}^{d}\left(\left\|a_{j}\right\|_{\rho}-\left|a_{j}(1)\right|\right) z^{j} .
$$

If $d=1$ and $a_{1}, a_{0} \in \mathcal{A}_{\rho}$ for some $\rho \in \mathbb{R}$, then $P(z)=0$ and

$$
Q(z)=\frac{1}{\left|a_{1}(1)\right|}\left(\left\|a_{0}\right\|_{\rho}-\left|a_{0}(1)\right|+\left(\left\|a_{1}\right\|_{\rho}-\left|a_{1}(1)\right|\right) z\right),
$$

where we suppose that $a_{1}$ is not a constant coefficient, because the constant coefficient case is trivial. So (2.6) becomes

$$
2^{\rho-r} \leq \frac{x_{0}\left|a_{1}(1)\right|}{\left\|a_{0}\right\|_{\rho}-\left|a_{0}(1)\right|+\left(\left\|a_{1}\right\|_{\rho}-\left|a_{1}(1)\right|\right)\left(\left|z_{0}\right|+x_{0}\right)},
$$

where $z_{0}=-a_{0}(1) / a_{1}(1)$. Analyzing (2.10), we consider two cases:

First case: $2\left|a_{1}(1)\right| \leq\left\|a_{1}\right\|_{\rho}$. Then we fix any $r$ satisfying

$$
r>\rho-\frac{1}{\log 2} \log \left(\frac{\left|a_{1}(1)\right|}{\left\|a_{1}\right\|_{\rho}-\left|a_{1}(1)\right|}\right),
$$


and take

$$
x_{0}:=\max \left\{\frac{|g(2)|}{2^{r}}, \frac{2^{\rho-r}\left(\left\|a_{0}\right\|_{\rho}-\left|a_{0}(1)\right|+\left(\left\|a_{1}\right\|_{\rho}-\left|a_{1}(1)\right|\right)\left|z_{0}\right|\right)}{\left|a_{1}(1)\right|-2^{\rho-r}\left(\left\|a_{1}\right\|_{\rho}-\left|a_{1}(1)\right|\right)}\right\},
$$

where $g(2)=\frac{a_{1}(2) a_{0}(1)-a_{0}(2) a_{1}(1)}{a_{1}(1)^{2}}$ is defined by (2.9).

Second case: $2\left|a_{1}(1)\right|>\left\|a_{1}\right\|_{\rho}$. Then

$$
r=\rho, \quad x_{0}:=\max \left\{\frac{|g(2)|}{2^{\rho}}, \frac{\left\|a_{0}\right\|_{\rho}-\left|a_{0}(1)\right|+\left(\left\|a_{1}\right\|_{\rho}-\left|a_{1}(1)\right|\right)\left|z_{0}\right|}{2\left|a_{1}(1)\right|-\left\|a_{1}\right\|_{\rho}}\right\} .
$$

Note that $r=\rho$ in (2.13) can also be derived as follows: Let $A_{1}(s)=\sum_{n=1}^{\infty} a_{1}(n) n^{-s}$ be the Dirichlet series for the complex variable $s \in \mathbb{C}$ with $\operatorname{Re} \geq \rho$. Then $\left|A_{1}(s)\right| \geq$ $2\left|a_{1}(1)\right|-\left\|a_{1}\right\|_{\operatorname{Re}} \geq 2\left|a_{1}(1)\right|-\left\|a_{1}\right\|_{\rho}>0$. Hence [7] implies that $a_{1}^{-1} \in \mathcal{A}_{\rho}$ and then $g=-a_{1}^{-1} * a_{0} \in \mathcal{A}_{\rho}$.

Summarizing, we arrive at the following result.

Theorem 2.2. Let $a_{1}, a_{0} \in \mathcal{A}_{\rho}$ for some $\rho \in \mathbb{R}$ and let $a_{1}$ be a nonconstant coefficient satisfying $a_{1}(1) \neq 0$. Then a unique solution $g \in \mathcal{A}$ of $a_{1} * g+a_{0}=0$ belongs to $g \in \mathcal{A}_{r}$ and $\|g\|_{r} \leq \frac{\left|a_{0}(1)\right|}{\left|a_{1}(1)\right|}+x_{0}$, where $r, x_{0}$ are given either by $(2.11)$ or (2.12) when $2\left|a_{1}(1)\right| \leq\left\|a_{1}\right\|_{\rho}$, or by (2.13) when $2\left|a_{1}(1)\right|>\left\|a_{1}\right\|_{\rho}$.

More general systems of convolution equations are studied in [5], which need not be polynomial. But since computations like the above seem to be rather awkward for such systems, we omit them in this note. We give a rather simple example in the next section.

\section{Quadratic equations}

In this section, we study the quadratic equation

$$
g * g=a, \quad a(1)=1, \quad\|a\|_{\infty}:=\sup _{n \in \mathbb{N}}|a(n)|<\infty .
$$

We also suppose that $a$ is not a constant coefficient, because the constant coefficient case is trivial. Since $a(1)=1 \neq 0$, we can use Theorem 2.1 and Remark 2.1 for (3.1). An interesting discussion on the solvability of (3.1) and the more general polynomial equations $g^{* d}=a, a \in \mathcal{A}$, is given in [5].

For any $\rho>1$ we compute

$$
\|a\|_{\rho} \leq\|a\|_{\infty} \sum_{n=1}^{\infty} n^{-\rho} \leq\|a\|_{\infty}\left(1+\int_{1}^{\infty} x^{-\rho} d x\right)=\frac{\rho}{\rho-1}\|a\|_{\infty} .
$$

Also using Remark 2.1 we get

$$
\begin{aligned}
& \left|z_{0}\right|=|g(1)|=\sqrt{|a(1)|}=1, \quad|g(2)|=\frac{|a(2)|}{2|g(1)|} \leq\|a\|_{\infty} / 2, \\
& P(z)=z^{2} / 2, \quad Q(z)=\left(\|a\|_{\rho}-1\right) / 2 .
\end{aligned}
$$

So $(2.7)$ gives

$$
M_{0}=\max _{z \in[0, \infty)} \frac{\left(2 z-z^{2}\right)}{\|a\|_{\rho}-1}=\frac{1}{\|a\|_{\rho}-1}, \quad x_{0}=1,
$$

and by (2.8) we can take any $r_{0}$ such that

$$
r_{0} \geq \max \left\{\frac{\log (|a(2)| / 2)}{\log 2}, \rho+\max \left\{0, \frac{\log \left(\|a\|_{\rho}-1\right)}{\log 2}\right\}\right\} .
$$


Hence according to (3.2) we take

$$
r_{0}=\max \left\{\frac{\log \left(\|a\|_{\infty} / 2\right)}{\log 2}, \rho+\frac{1}{\log 2} \max \left\{0, \log \left(\frac{\rho\left(\|a\|_{\infty}-1\right)+1}{\rho-1}\right)\right\}\right\} .
$$

Now fixing $\|a\|_{\infty} \geq 1$ and varying $\rho>1$, we intend to find the smallest value of $r_{0}$ in (3.3). We consider two cases:

First case: $\|a\|_{\infty} \geq 2$. Then it holds that

$$
\frac{\rho\left(\|a\|_{\infty}-1\right)+1}{\rho-1} \geq\|a\|_{\infty}-1 \geq\|a\|_{\infty} / 2 \geq 1 .
$$

Consequently, (3.3) possesses the form

$$
r_{0}=F_{\|a\|_{\infty}}(\rho):=\rho+\frac{1}{\log 2} \log \left(\frac{\rho\left(\|a\|_{\infty}-1\right)+1}{\rho-1}\right) .
$$

It is easy to verify that $F_{\|a\|_{\infty}}(\rho)$ is strictly convex on $(1, \infty)$, i.e. $F^{\prime \prime}\|a\|_{\infty}(\rho)>$ $0 \forall \rho>1$. So the function $F_{\|a\|_{\infty}}(\rho)$ has the unique global minimum on $(1, \infty)$ achieved at a unique $\rho_{0}>1$ solving $F_{\|a\|_{\infty}}^{\prime}\left(\rho_{0}\right)=0$. But for a general $\|a\|_{\infty}$, the explicit formula for this global minimum $F_{\|a\|_{\infty}}\left(\rho_{0}\right)$ is ugly and long, so we do not present it here. On the other hand, we computed for $\|a\|_{\infty}=10$ that $\rho_{0} \doteq 1.82706$ and $F_{10}\left(\rho_{0}\right) \doteq 6.22562$.

Second case: $\|a\|_{\infty}<2$. Then (3.3) possesses the form

$$
r_{0}= \begin{cases}\rho & \text { for } \quad \rho \geq \frac{2}{2-\|a\|_{\infty}}, \\ F_{\|a\|_{\infty}}(\rho) & \text { for } 1<\rho \leq \frac{2}{2-\|a\|_{\infty}} .\end{cases}
$$

Since again for a general $\|a\|_{\infty}$ the explicit formula for the global minimum of (3.4) is ugly and long, we do not present it here. We concentrate on the case $\|a\|_{\infty}=1$. Then (3.4) becomes

$$
r_{0}= \begin{cases}\rho & \text { for } \rho \geq 2, \\ F_{1}(\rho)=\rho-\frac{\log (\rho-1)}{\log 2} & \text { for } 1<\rho \leq 2 .\end{cases}
$$

We can easily check that the piecewise smooth function in (3.5) has a unique global minimum 2 on $(1, \infty)$ at $\rho_{0}=2$.

Summarizing from Theorem 2.1 and Remark 2.1 we obtain the following result.

Corollary 3.1. Let a be a nonconstant coefficient. If $\|a\|_{\infty}=1$ then the only two solutions $g_{ \pm} \in \mathcal{A}$ of (3.1) with $g_{ \pm}(1)= \pm 1$, respectively, belong to $\mathcal{A}_{2}$ and $\left\|g_{ \pm}\right\|_{2} \leq 2$. If $\|a\|_{\infty}=10$, then $g_{ \pm} \in \mathcal{A}_{r_{0}}$ and $\left\|g_{ \pm}\right\|_{r_{0}} \leq 2$ for $r_{0} \doteq 6.22562$.

According to [5, Corollary 2], we obtain $g_{-}=-g_{+}$in Corollary 3.1. Similar numerical results hold for other numerical values of $\|a\|_{\infty}$.

\section{REFERENCES}

[1] H. BILleR: Analyticity and naturality of the multi-variable functional calculus, TU Darmstadt Preprint 2332, 2004; http:wwwbib.mathematik.tu-darmstadt.de/Math-Net /Preprints/Listen/files/2332.ps.gz.

[2] T. Carroll And A. A. Gioia: Roots of multiplicative functions, Compositio Mat. 65 (1988), 349-358. MR932075 (89c:11007)

[3] M. J. Cohen: Advanced problem 5293, Amer. Math. Monthly 72 (1965), 555; Solution, ibid 73 (1966), 553-554.

[4] P.-O. DehaYe: On the structure of the group of multiplicative arithmetical functions, Bull. Belgian Math. Soc. 9 (2002), 15-21. MR1905645 (2003d:11009) 
[5] J. Glöckner, L. G. LuCht And Š. PorubskÝ: Solutions to arithmetic convolution equations, Proc. Amer. Math. Soc., to appear. MR2286069

[6] P. HaukKanen: Arithmetical equations involving semi-multiplicative functions and the Dirichlet convolution, Rend. Mat. Appl., VII. Ser. 8 (1988), 511-517. MR1032719 (90k:11007)

[7] P. Hewitt and J. H. Williamson: Note on absolutely convergent Dirichlet series, Proc. Amer. Math. Soc. 8 (1957), 863-868. MR0090680 (19:851b)

[8] Š. PoRUBSKÝ: Structure of the group of quasi-multiplicative arithmetical functions, Acta Arith. Paed. Agiensis, Sect. Math. 30 (2003), 133-145. MR2054723 (2005b:11006)

[9] M. V. SubbaraO: A class of arithmetical functions, Nieuw Archief voor Wiskunde (3) 15 (1967), 211-217. MR0223293 (36:6341)

Department of Mathematical Analysis and Numerical Mathematics, Comenius University, Mlynská dolina, 84248 Bratislava, Slovakia; and Mathematical Institute, Slovak Academy of Sciences, Štefánikova 49, 81473 Bratislava, Slovakia

E-mail address: Michal.Feckan@fmph.uniba.sk 